\title{
Las concepciones de los padres sobre el desarrollo: Teorías personales o teorías culturales
}

\author{
BEATRIZ TRIANA \\ Universidad de la Laguna

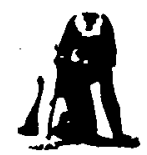 \\ Resumen
}

Este artículo ofrece un modelo teórico para explicar el origen de las concepciones de los padres sobre la educación y el desarrollo, y cómo éstas se plasman en la mente de los padres. Se postula que la cultura ofrece un amplio bagaje de concepciones que se transmiten mediante las interacciones que establecen los sujetos en los contextos sociales próximos. No obstante, el papel de la cultura queda regulado por los mecanismos de elaboración de la información que caracterizan el procesamiento bumano. Esto se demuestra al analizar, a partir de un conjunto de teorias de gran raigambre cultural, las mezclas coberentes de teorias que asumen los padres. Los objetivos que se persiguen son: a) Elaborar un cuestionario para estudiar las teorias; y b) analizar el papel de los contextos sociales próximos en la selección de las teorias que asumen los padres, destacando el nivel educativo y profesional de los padres asi como la zona donde residen, como variables determinantes del tipo de teorials que éstos seleccionan.

Palabras clave: Concepciones, Educación, Desarrollo, Cultura, Contextos sociales próximos, Procesamiento bumano.

\section{Parent's conceptions about development: Personal or cultural theories}

\section{Abstract}

This paper proposes a theoretical model aiming to explain the sources of parents' conceptions on education and the way they are represented in their minds. We assume that culture offers a wide background of conceptions which are transmitted through the interactions which subjects establish in close social context. However, the role of culture is regulated by the information processing mechanisms which characterizes buman processing. This is shown when, on the basis of a set of theories with deep cultural roots, the coberent mixture of theories which parents assume were analysed. The objetives to follow were:

a) To devise a questionnaire to study these theories.

b) To analyse the role of close social contexts in the selection of theories which parents assume, underlying parents' academic and professional background together with the area in which they live as determinant variables of the type of theory they choose.

Keywords: Conceptions, Education, Development, Culture, Close social context, Human processing.

Agradecimientos: La presente investigación se ha desarrollado bajo el patrocinio de la CICYT (Proyecto n. ${ }^{\circ}$ PB 86-0601), dirigido por Maria José Rodrigo López, de cuyo equipo investigador forma parte la autora.

Dirección de la autora: Dpto. de Psicología Educativa, Evolutiva y Psicobiología. Universidad de la Laguna. Tenerife.

Original recibido: Marzo, 1990. Revisión recibida: Abril, 1990. Aceptado: Mayo, 1990 


\section{INTRODUCCION}

El estudio de las relaciones entre padres e hijos ha sufrido un amplio giro en las dos últimas décadas. La concepción de los padres como sujetos reactivos ante la conducta manifiesta de sus hijos, ha dado paso a una nueva imagen en la que se les concibe como seres pensantes que se enfrentan a la tarea educativa equipados con un amplio bagaje de conocimientos sobre la infancia (v.g.: cuál es su naturaleza, cómo evolucionan, cuáles son las causas de su conducta y sus posibilidades de entrenamiento, etc.). Por esta razón, muchos autores dirigen actualmente su interés hacia el estudio de los orígenes y determinantes de las teorías implícitas de la educación que sostienen los padres (Triana y Rodrigo, 1985), qué relación guardan con las prácticas de crianza que utilizan (McGillicuddy-De Lisi, 1982; Sameroff y Feil, 1985; Palacios, 1986), y en última instancia, qué repercusiones tienen sobre el desarrollo de los niños (Brooks-Gunn, 1985; Schaefer y Edgerton, 1985, entre otros). Este artículo versa sobre el primer aspecto, esto es, cuál es el origen de las concepciones de los padres sobre la infancia, y lo que es más importante, cómo se plasman estas ideas en la mente del hombre de la calle.

Goodnow (1987) ha recogido los dos planteamientos teóricos que se han utilizado para abordar este tema. Por un lado, el enfoque psicologista que asume que las teorías son construcciones personales que se derivan del cúmulo de experiencias directas de los sujetos, sirviendo como ejemplo los «constructos personales» de Kelly (1955). Según esta orientación, cabría esperar la existencia de tantas teorías sobre el desarrollo y la educación como padres a estudiar. Por su parte, el enfoque sociologista asume que es la cultura y la sociedad en la que se desenvuelve el hombre de la calle, quienes determinan sus concepciones. Según esta perspectiva, las teorías se definen como «mensajes culturales» o «conocimiento recibido» (Goodnow, 1987), lo que supondría la existencia de diferentes concepciones sobre el desarrollo y la educación sólo apreciables en los contrastes transculturales, encontrándose una gran homogeneidad en las concepciones dentro de un mismo ámbito cultural.

Es evidente que los grupos sociales contribuyen a normativizar el tipo de experiencias que reciben sus miembros. No obstante, asumir la tesis de una mera transmisión culturàl (v.g.: las representaciones colectivas de Moscovici), sería negar el poder de selección y construcción de la información que caracteriza el funcionamiento cognitivo humano. Como alternativa a las dos posiciones anteriores, existe una tercera que se podría denominar "socioconstructivista", que postula que las teorías implícitas son fruto de una construcción personal a partir de rasgos o contenidos culturales (Rodrigo, 1985). Así, la cultura juega un papel primordial sirviendo como sustento de ideas y experiencias para la elaboración de las teorías implícitas. Del mismo modo, cumple una segunda función de transmisión de estos contenidos, propiciando las interacciones que establecen las personas en los contextos sociales próximos. En estos contextos es donde se activan los filtros interpretativos y valorativos que caracterizan a cada grupo social. Por último, cabe decir que la influencia de la cultura queda regulada por la construcción individual que realiza el hombre de la calle a partir de la informa- 
ción recibida, y es gracias a esta elaboración como se producen las diferencias individuales.

En un estudio previo, Triana y Rodrigo (1985) rastrearon el origen de una serie de concepciones de los padres sobre el desarrollo y la educación tomando como guía algunas teorías científicas sobre la infancia. Para ello utilizaron diferentes procedimientos metodológicos con el objeto de apresar tanto el contenido cultural de las concepciones de los padres como su carácter de representación interna e individual. Así utilizaron técnicas de investigación bistoriográficas para captar las regularidades culturales, seleccionando las teorías de la educación que han tenido mayor relevancia (v.g.: Homúnculo, Nurturista, Roussoniana, Ambientalista, Innatista, Voluntarista y Constructivista); la técnica de brainstorming para determinar el conjunto de enunciados que reflejaban las ideas principales de cada teoría; y procedimientos normativos para analizar su carácter de esquema de conocimiento. En este estudio los sujetos categorizaron de forma coherente cada una de las teorías analizadas, incluso las más ancestrales, lo cual muestra que las teorías de origen cultural tienen cierta entidad en la mente de los padres. Asimismo, la estructura interna de las teorías se rige por principios de tipicidad, al igual que las categorías y otros esquemas de conocimiento.

No obstante, es evidente que si bien los padres reconocen las diferentes teorías que han ido surgiendo a lo largo de la historia, también es cierto que a la hora de tomarlas como guía para su quehacer educativo, seleccionan sólo algunas de ellas. ¿Qué factores organizan esta selección? Es ahora 'cuando toman especial relevancia los contextos interactivos próximos, ya que éstos favorecen la exposición de los padres a conjuntos de experiencias más acordes con determinadas teorías frente a otras. Estas experiencias no sólo se plasmarían en la memoria con mayor intensidad, sino que también su activación en situaciones recurrentes las convertirían en conjuntos de conocimiento con un carácter más accesible para los padres.

Es importante plantearse si las teorías que asumen los padres como propias tienen la misma entidad funcional que el resto de teorías que conocen. En este sentido, Sigel (1985) distingue entre creencias (teorías resumidas) y. conocimiento (teorías conocidas). Aunque ambas son construcciones de la realidad, la diferencia estriba en el valor de verdad que se le atribuye a las primeras frente a las segundas. A nuestro entender, existen además otros aspectos diferenciadores como puede ser el carácter más esquemático del conocimiento frente a la flexibilidad de las creencias, que se manifiesta en la posibilidad de mezclar ideas de varias teorias, con la condición de que éstas sean siempre coherentes. Asimismo, las creencias se procesan con carácter preferente y actúan como guía para la realización de inferencias, e incluso, para la propia acción.

En los estudios que se presentan en este artículo se persiguen dos objetivos. El primero de ellos es construir un instrumento que permita estudiar las teorías educativas de los padres, en su carácter de creencias, comparando además la eficacia de dos procedimientos de análisis diferentes, las técnicas normativas (empleada por Triana y Rodrigo, 1985) y las psicométricas. El segundo objetivo es comprobar la influencia de los contextos interactivos próximos, evaluados mediante diversas variables demográficas, en la determinación de las teorias que se atribuyen a los padres. 


\section{PRIMER ESTUDIO: ELABORACION DEL CUESTIONARIO DE TEORIAS IMPLICITAS SOBRE EL DESARROLLO Y LA EDUCACION}

Un aspecto de gran interés dentro de la Psicología ha sido la búsqueda de instrumentos de evaluación que permitan al investigador abordar los temas de estudio de forma objetiva. Sin embargo, ello no siempre es posible como lo demuestran las críticas de que han sido objeto las técnicas de observación directa, las entrevistas o incluso los cuestionarios. Por ejemplo Sigel (1986), en el caso de los cuestionarios, resalta, entre otros inconvenientes, el tener que enfrentar a los sujetos a una estructura categorial preestablecida por el propio investigador; el ignorar los efectos que ejerce la deseabilidad social en las respuestas; la pluralidad de los temas que se pretenden abarcar en los estudios, etc. Ante estas críticas, cabría cuestionar la utilidad de estos instrumentos, o bien considerar la posibilidad de que los inconvenientes se deriven de los sesgos establecidos en el proceso de elaboración. A nuestro parecer, la segunda asunción es la más acertada, y en consecuencia, el esfuerzo de los investigadores debe ir dirigido a corregir los sesgos anteriormente citados.

Concretamente, en el cuestionario de teorías implícitas que se pretende construir, la información que sirve de base para su elaboración no la desarrolla a priori el investigador, sino que se recoge a partir de una muestra representativa de las personas que serán posteriormente sujetos de estudio. Así se registran las expresiones que ellas comúnmente emplean para refe. rirse al tema, y los aspectos más relevantes del mismo vistos desde su perspectiva. En nuestra opinión, todo ello facilita la comprensión y correcta evaluación de las ideas cuando se realiza la recogida de datos. Con esta finalidad, en un estudio previo se utilizó una metodología que combina diferentes técnicas como son los análisis historiográficos, las técnicas de brainstorming y los estudios normativos (Triana y Rodrigo, 1985). En este estudio, a través de las técnicas normativas se pudo conocer cuáles son los enunciados que mejor definen cada una de las teorías de la educación en estudio, con el fin de utilizarlas para la elaboración del cuestionario.

Ahora bien, la necesidad de construir un instrumento psicométrico que cumpla los requisitos de fiabilidad y validez, hace necesaria la utilización de técnicas estadísticas para su selección. Por ello, en la presente investigación se utilizará el conjunto de enunciados obtenidos en el estudio anteriormente citado, como fuente de datos para la construcción del cuestionario psicométrico. Con este fin, una muestra de sujetos évaluará el conjunto de enunciados conforme a su grado de acuerdo con sus propias ideas. A partir de estas puntuaciones se realizarán diversos análisis factoriales exploratorios, para comprobar si las teorías constituyen verdaderos factores independientes. De ser así, las variables que mejor conformen la estructura factorial, pasarán a constituir el cuestionario definitivo para estudiar las teorías implícitas de la educación. La investigación consta de dos fases, la primera de tipo exploratorio y la segunda para lograr una confirmación de los resultados obtenidos. 
Fase I. Primer estudio para la configuración del cuestionario de las teorías implícitas sobre la educación

Se pretendía seleccionar de una amplia lista de enunciados, aquellos que siguiendo técnicas psicométricas demuestren ser los más idóneos para formar el cuestionario de las teorías implícitas sobre la educación y el desarrollo.

\section{Método}

Sujetos: En este estudio participó una muestra de 261 estudiantes, de ambos sexos, con una edad media de 23,2 años, que cursaban estudios de Magisterio y Puericultura.

Material y procedimiento: Se elaboró un instrumento en el que los sujetos debían cumplimentar, en primer lugar, los datos personales y leer las instrucciones para su realización, junto con tres ejercicios cumplimentados a modo de ejemplos. A continuación se presentaba el conjunto de los 130 enunciados seleccionados en la investigación de Triana y Rodrigo (1985), relativos a diferentes teorías. Con el fin de facilitar la tarea atribucional, se encabezó cada frase con giros autoreferenciales como los siguientes: "En mi opinión», «es necesario», «yo...», «creo que», «debemos», etc. Teniendo en cuenta el amplio número de items a evaluar en el cuestionario, se invirtió el significado del $25 \%$ de los enunciados de cada teoría, con el fin de evitar que los sujetos realizaran la prueba de forma mecánica, y forzarles, en la medida de lo posible, a un análisis cuidadoso de cada uno de ellos. El formato de respuesta consistió en presentar el conjunto de proposiciones (en el margen izquierdo de la hoja) con sus correspondientes escalas numéricas (en el margen derecho), donde los sujetos reflejaban sus juicios.

En las instrucciones se hizo gran hincapié en señalar a los sujetos que se trataba de un ejercicio en el que no existían aciertos ni errores. Su tarea consistía en expresar su grado de acuerdo con las ideas que refleja cada enunciado, evaluándolas mediante una escala de «0 a 4» puntos (desacuerdo a acuerdo). Para facilitar la familiarización de los sujetos con la escala, se ofrecían los siguientes ejemplos resueltos, que mostraban la manera de puntuar las frases según se tratara de un caso de acuerdo alto, intermedio o bajo.

1) ... Supongamos que usted estuviera muy de acuerdo con la siguiente frase: «Nada ni nadie puede cambiar al niño desde que nace». Su puntuación sería de «4» puntos.

2) ... Supongamos ahora que usted no está ni en total acuerdo ni en total desacuerdo con la siguiente frase: "Los niños son una mezcla de reacciones adultas e infantiles». Su puntuación sería «2» puntos.

3) ... Supongamos, por último, que usted está en total desacuerdo con la siguiente frase: «El niño es tan poco capaz que todo lo que sabe ha sido aprendido por imitación de sus padres». Su puntuación en esta frase sería de «0» puntos.

El tiempo de ejecución de la tarea era limitado y se animó a los sujetos a-preguntar siempre que tuvieran alguna duda. 


\section{Resultados y conclusiones}

El objetivo de este estudio era construir, a partir de las ideas recogidas en los brainstormings, un instrumento de medida perfectamente validado que nos permitiera estudiar las diferentes teorías sobre el desarrollo y la educación sustentadas por los padres.

En primer lugar, se aplicó el programa FREQUENCIES del paquete estadístico SPSS para seleccionar los items que presentaban una distribución normal en los juicios de los sujetos y cuyo índice de asimetría estuviese comprendido entre los valores $(-3,+3)$. De este modo, el $28,46 \%$ de los enunciados del cuestionario quedaron eliminados por no tener valor diagnóstico, ya que la mayoría de los sujetos tendían de forma unánime a compartir sus ideas o a rechazarlas.

El conjunto de enunciados restantes fue estudiado en reiterados análisis factoriales en los que se observó una pauta fija de resultados. Por un lado, los items de la teoría del homúnculo presentaban pesos factoriales bajos en diferentes factores. En los estudios normativos (Triana y Rodrigo, 1985), los enunciados homunculistas eran perfectamente categorizados por los sujetos como pertenecientes a esa teoría, lo cual implica que forma parte del marco conceptual de los sujetos. No obstante, en los estudios atribucionales la teoría del homúnculo se presenta como una de las más rechazadas. Este hecho no es extraño ya que una persona puede conocer una determinada teoría, pero no compartirla en absoluto. En efecto, parece que la visión estática del infante que ofrece esta teoría, choca con cualquier visión evolucionista del desarrollo que postule la existencia de cambios sustanciales entre el niño y el adulto. En consecuencia, aun reconociendo su entidad representacional, no es útil mantenerla de cara a estudiar su distribución en la población.

Por otra parte, la teoría roussoniana se presentaba compartiendo un mismo factor con la teoría ambientalista, aunque los pesos de la primera eran más bajos que los de la segunda. Este segundo resultado nos llevó a eliminar también la teoría roussoniana, ya que al parecer ambas medían lo mismo. Una posible explicación de este resultado puede ser que aunque los padres son conscientes de la influencia que puede tener el ambiente sobre el niño (aspectos compartidos con la teoría ambientalista), no llegan a afirmar que toda experiencia social sea nociva (ideas más propiamente roussonianas), máxime cuando ellos se sienten los principales agentes de socialización de sus hijos. Este conflicto puede llevarles con frecuencia a tomar posturas moderadas ante esta teoría.

Continuamos entonces los análisis factoriales con las cuatro teorías restantes. Así se obtuvo una estructura factorial en la que un conjunto de seis proposiciones de cada teoría correlacionan, aunque de forma moderada (alrededor de .50), respecto a un único factor. De este modo, quedó constituida una matriz de cuatro factores, cada uno de los cuales corresponde a una de las teorías en estudio (esta matriz no la presentamos por la cuantía de datos que supone esta investigación, considerando de mayor interés la correspondiente al estudio siguiente).

Antes de finalizar este apartado quisiéramos mencionar un resultado a nuestro entender muy interesante. A partir de los estudios normativos (Triana y Rodrigo, 1985), se había comprobado que las teorías implícitas 
son conjuntos de ideas organizadas según un continuo de tipicidad. Así, las más prototípicas concentran información altamente representativa de esa teoría, las moderadamente típicas contienen información referente tanto a esa teoría como a cualquier otra, y las de baja tipicidad hacen referencia a ideas propias de alguna otra teoría. Extrapolando estos resultados a los ahora alcanzados a través de la metodología psicométrica, se puede observar que los enunciados se distribuyen en la estructura factorial adoptando una pauta similar a la anteriormente citada. Esto es, aquellos enunciados que mejor conforman la estructura factorial (obtienen pesos altos y sólo con respecto al factor correspondiente a la teoría) suelen ser los de alta tipicidad, los de tipicidad moderada correlacionan bajo y lo hacen respecto a varios factores; y por último, los de baja tipicidad suelen correlacionar alto con algún factor que responde a otra teoria alternativa. Los resultados reflejan la posibilidad de un fructífero complemento entre dos metodologías diferentes para tal fin: la normativa y la psicométrica.

No obstante, no existe una correspondencia exacta entre la estructura de tipicidad y la solución factorial. Como ya hemos indicado, no todas las proposiciones prototípicas son las que mejor comportamiento estadístico - presentan. Quizá la mejor explicación de este resultado, así como del hecho de que se obtengan pesos factoriales y comunalidades moderadas, es que se trata de teorías que comprenden conjuntos de ideas a veces bastante complejas, lo cual se presta a una mayor variabilidad en las puntuaciones dadas por los sujetos ante cada item, que si estuviéramos trabajando con algún otro tipo de esquema más concreto como pudiera ser el de las categorias naturales.

\section{Fase II. Segundo estudio para la configuración del cuestionario de las teorías implícitas}

La segunda fase de este estudio consistió en poner a prueba el primer esbozo de cuestionario obtenido en la fase anterior, con el fin de comprobar si la estructura factorial se mantiene estable, con una nueva muestra de sujetos, y en su caso, elaborar el cuestionario definitivo con los enunciados seleccionados.

\section{Método}

Sujetos: Participaron en este estudio un total de 209 sujetos de los cuales el $75 \%$ pertenecía al sexo femenino, con una edad media de 21,2 años, estudiantes del segundo y tercer curso de Psicología, y tercer curso de Pedagogía o Magisterio.

Material y procedimiento: Se elaboró un cuadernillo con el conjunto de enunciados seleccionados en la fase anterior, a la que añadimos 3 items más para cada una de las teorías, eligiéndolos según el criterio de que fueran también de alta tipicidad, aleatorizando su presentación. En esta ocasión no se presentaron enunciados con información invertida, y se modificó la escala evaluativa que toma ahora un rango de $" 0$ a 8 " puntos, con el fin de alcanzar una mayor precisión en los análisis estadísticos, lo que se consigue ampliando el margen de puntuaciones.

El formato de este nuevo cuestionario era similar al anteriormente utilizado, y por ello no pasamos a describirlo. Nuevamente se trataba de una 
tarea en la que los sujetos debían juzgar su grado de acuerdo con las ideas acerca de la educación reflejadas en los diferentes enunciados, tomando como referencia la escala evaluativa que se les explicaba en las instrucciones.

\section{Resultados}

El objetivo primordial que perseguíamos era comprobar si los resultados obtenidos en la fase anterior se verificaban de nuevo con otra muestra de sujetos y no respondian a un mero artefacto estadístico. Para ello se realizaron varios análisis factoriales utilizando diferentes combinaciones de items. Al eliminar algunos enunciados que obtuvieron pesos factoriales bajos, se consiguió la matriz factorial que presentamos a continuación.

\section{TABLA I}

Matriz factorial de las variables rotadas

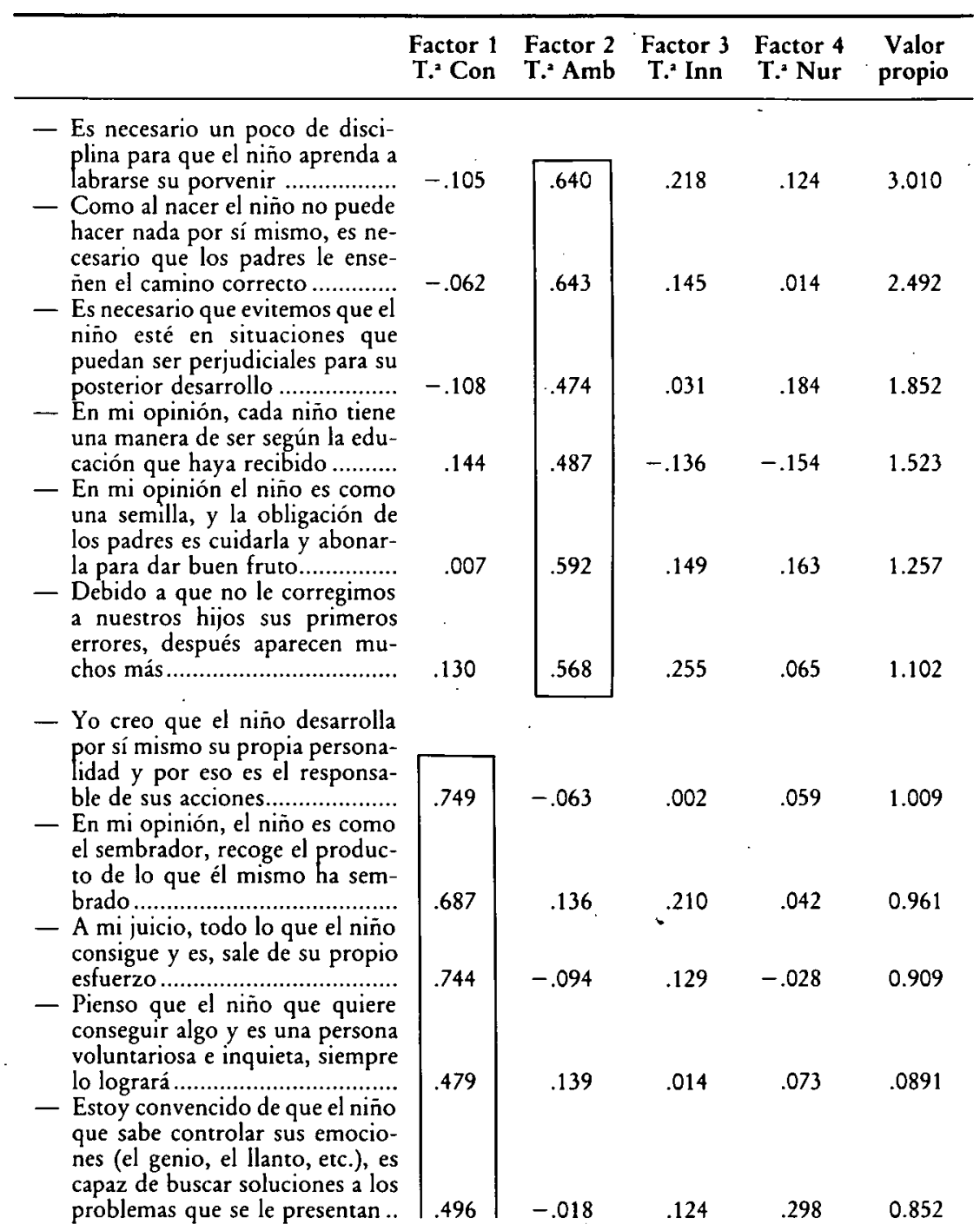


TABLA I

(Continuación)

Factor 1 Factor 2 Factor 3 Factor 4 Valor

T.' Con T.' Amb T.2 Inn T.' Nur propio

- Creo que cada niño construye su propio futuro

\begin{tabular}{l|lllll}
.721 & -.046 & -.052 & .021 & 0.765
\end{tabular}

- Al niño le debemos dejar que actúe con libertad, porque es muy difícil que modifiquemos lo que viene determinado por la herencia comportarse del niño la ha heredado de sus padres ................

- Debido a la herencia, desde que nace un bebé podemos observar muchas características propias de sus padres

- Debido a que el niño nace con una forma de ser heredada de sus padres, es muy difícil que le podamos cambiar.

.... Yo creo que la herencia determina el desarrollo posterior del niño.

.

\begin{tabular}{|c|c|c|c|c|}
\hline .199 & .072 & .477 & -.088 & 0.728 \\
\hline .067 & .283 & .524 & .119 & 0.700 \\
\hline .001 & .158 & .655 & .198 & 0.677 \\
\hline-.073 & .231 & .630 & .028 & 0.666 \\
\hline .115 & .029 & .452 & .172 & 0.601 \\
\hline-.010 & -.049 & .608 & .013 & 0.544 \\
\hline-.071 & .040 & .614 & .236 & 0.540 \\
\hline-.022 & .070 & .192 & .496 & 0.515 \\
\hline-.049 & .093 & .233 & .561 & 0.495 \\
\hline-.009 & . 207 & -.348 & .597 & 0.439 \\
\hline .295 & .031 & 1117 & .664 & 0.430 \\
\hline .074 & .025 & .070 & .625 & 0.401 \\
\hline .054 & .306 & .011 & .530 & 0.389 \\
\hline .162 & -.057 & .038 & .659 & 0.311 \\
\hline
\end{tabular}

- Debido a que los niños heredan nuestros fallos, debemos disculparles y no castigarles..............

- Es inútil que le demos instrucción al niño, porque cuando nace su desarrollo está programado

- Estoy convencido de que un niño enfermizo fracasa en cualquier tarea

- Debido a que la alimentación y la salud son los factores más importantes en el éxito de los ninos, nuestra educación debe ir encaminada en que coman mucho y no tengan enfermedades.

- Creo que es difícil enseñar a un niño si lo tienes mal alimentado

- Pienso que la salud y la buena alimentación permiten que el niño se desarrolle sin ningún problema

- Creo que cuando el niño no rinde en la escuela es porque no come lo suficiente.

- El niño tiene que tener salud para que lo podamos educar correctamente

- Pienso que los niños con buena alimentación no tendrán ningún problema a nivel de educación.

Contrastando la matriz que se obtuvo en la fase anterior, pudimos observar que ahora las variables ofrecen, en conjunto, pesos factoriales ligeramente más altos, así como un valor propio, esto es, los factores explican un mayor porcentaje de varianza de las variables. 
$\mathrm{Al}$ analizar los enunciados que han perdurado observamos que no existe la misma pauta en las diferentes teorías. Concretamente, tan sólo la teoría nurturista mantiene los mismos items y además, incorpora uno de los añadidos de alta tipicidad. La teoría innatista pierde el item que menos correlacionaba anteriormente con el. factor y se incorporan dos de los añadidos. Por último, en lo que respecta a las teorías ambientalista y constructivista, ambas pierden dos items que antes parecían ajustarse bien al factor, e incorporan otros dos de los añadidos. La posible explicación de estos resultados se deriva del propio fundamento estadístico de estos análisis, según el cual, la incorporación de una nueva variable o la supresión de alguna ya existente, afecta al conjunto de la estructura factorial.

A continuación realizamos un análisis factorial confirmatorio, mediante el programa LISREL, con el conjunto de los 26 items seleccionados. En él se obtuvo un índice de bondad de ajuste de la prueba de .735 . Ello implica que a pesar de los cambios, la estructura factorial obtenida con estos datos se ajusta o coincide con el modelo obtenido a partir de la muestra anterior.

\section{Discusión y conclusiones}

La segunda fase de este estudio ha tenido como objetivo comprobar, una vez más, si los conjuntos de enunciados de las teorías constituyen verdaderos factores independientes, coincidiendo su distribución con la estructura factorial obtenida anteriormente. Los resultados han confirmado nuevamente nuestra suposición, ya que hemos obtenido un conjunto de enunciados para cada teoría, que correlaciona, aunque moderadamente, con un único factor. Estos items pertenecen a lòs categorizados como altamente típicos en nuestras investigaciones anteriores (Triana y Rodrigo, 1985). Este hecho valida la eficacia de utilizar los estudios normativos como primer paso para configurar las teorías implícitas.

Además, hemos perfilado la selección definitiva de enunciados, obteniendo una estructura factorial más nítida que en la fase anterior, y que ha sido fundamentada mediante un análisis factorial confirmatorio. Las 26 ideas seleccionadas (ver Tabla I), pasarán a constituir el cuestionario definitivo de las teorías implícitas sobre el desarrollo y la educación.

Por último, quisiera señalar algunas de las ideas centrales que definen cada una de estas teorías:

Teoría constructivista: El desarrollo del niño depende de su voluntad y esfuerzo, ya que es el responsable de sus propias acciones. El tipo de práctica educativa que se deriva de esta teoría es el fomento del autocontrol.

Teoria ambientalista: El ambiente determina el desarrollo. Así, la disciplina de los padres adquiere gran importancia ya que asume que el niño es un ser pasivo que hay que modelar mediante las prácticas educativas.

Teoria innatista: El desarrollo viene determinado por la herencia. La disciplina reduce su importancia ya que los rasgos psicológicos difícilmente se pueden cambiar.

Teoria nurturista: El crecimiento físico es la fuerza que dirige el desarrollo. El interés de los padres se centra en la salud y la buena alimentación como factores cruciales para la educación y el éxito escolar.

Como se puede apreciar, este conjunto de enunciados que han sido seleccionados por el hombre de la calle como reflejo de cada una de las teo- 
rías en análisis, son versiones menos formales de las teorías científicas de las que se derivan. Ello no es extraño ya que los padres han elaborado este conocimiento en aprendizajes espontáneos (fundamentalmente basados en experiencias) con escaso grado de formalización.

\section{SEGUNDO ESTUDIO: EL PAPEL DE LOS CONTEXTOS INTERACTIVOS PROXIMOS EN LA DETERMINACION DE LAS CONCEPCIONES DE LOS PADRES}

El objetivo de esta investigación es estudiar las teorías implícitas que sostienen los padres sobre el desarrollo y la educación de sus hijos, y qué factores determinan su selección. El interés de su estudio radica en su poder interpretativo de la compleja realidad educativa, y su influencia en la aplicación de determinadas prácticas de crianza. En este sentido, determina el estudio educativo parental, y éste a su vez, influye en el nivel de funcionamiento cognitivo del niño (Sigel, 1985; Bacon y Ashmore, 1986; entre otros).

La pluralidad de las pautas educativas observadas en distintos padres puede explicarse aludiendo al carácter sociocultural de las teorías. En este sentido, la cultura ofrece todo un bagaje de ideas que han ido surgiendo a lo largo de nuestra historia. Ahora bien, son los contextos interactivos próximos los responsables de la selección que realizan los padres a la hora de atribuirse algunas de ellas como propias. Siguiendo este argumento, nuestro interés se dirige ahora hacia la concreción de cuáles son tales contextos.

Algunos autores han abordado este tema analizando indicadores que contribuyen a definir estos contextos. Por ejemplo, se ha estudiado cómo influye el factor generación en la transmisión de las teorías asumidas, aunque los resultados son algo contradictorios (Cashmore y Goodnow, 1985); otros autores señalan el papel de la pareja y las experiencias compartidas como contexto clave en el que se forman y modifican las teorías parentales (McGillicuddy-De Lisi, 1982); también el nivel educativo y socioeconómico de los padres, así como la pertenencia a un grupo étnico, parece estar altamente relacionado con sus ideas acerca de la educación y el desarrollo (Skinner, 1985; Palacios, 1986; entre otros); asimismo, el número de hijos que componen la familia parece estar relacionado con las teorías compartidas por los padres (Knight, 1983), etc.

En nuestro estudio seguimos una línea de investigación similar a la de estós autores, no obstante hay que señalar dos claras diferencias. En primer lugar, se trata de teorías generales de claro raigambre cultural, y en segundo lugar, la metodología que utilizamos se fundamenta en técnicas psicométricas. Concretamente, partimos de las cuatro teorías (ambientalista, innatista, constructivista y nurturista) seleccionadas en el estudio anterior, con el fin de que una amplia muestra de padres las evalúen conforme al grado de acuerdo con su propio modelo educativo, y averiguar así sus teorías evolutivas. Para comprobar la influencia de los contextos sociales próximos en la configuración de las teorías que asumen los padres, se tomaron como indicadores de tales contextos el sexo, la edad, la profesión, el nivel de estudios, la zona en la que residen (urbana-rural) y el número de hijos. 


\section{Método}

Sujetos: En este estudio participó una muestra de 371 padres, de zona rural y urbana, de los que el $69,5 \%$ eran madres. Dentro de este grupo se engloban 72 parejas. El intervalo de edad comprendía sujetos entre los 18 y 57 años, con una media de 36,4. El $55 \%$ de la muestra tenían 1 ó 2 hijos, y el resto más de 2 . Respecto al nivel de estudios y la profesión, el porcentaje de sujetos para cada nivel de las variables no estaba tan bien distribuido, pero permitía realizar los análisis estadísticos pertinentes.

Diseño: El diseño puede describirse técnicamente como un diseño factorial completo de factores cruzados $2 \times 2 \times 2 \times 3 \times 4 \times 2 \times 4$, donde los seis primeros factores, sexo (masculino/femenino), edad (menor de 36 años/igual o superior a 36 años), número de hijos (hasta 2 hijos/más de 2), nivel profesional (bajo/medio/alto), nivel de estudios (sin estudios/primaria/bachiller superior o carrera de grado medio/estudios superiores) y zona en la que residen (rural/urbana) son intergrupo, mientras que el último factor, tipo de teoria (ambientalista/innatista/constructivista/nurturista) es intragrupo. Como se puede observar, las variables edad y número de hijos fucron dicotomizadas.

La variable dependiente fue el grado de acuerdo de las ideas de cada padre o madre con los diferentes enunciados.

Material y procedimiento: Se utilizó el cuestionario con el conjunto de los 26 enunciados seleccionados en el estudio anterior, aleatorizando su presentación. El formato era similar al utilizado en el estudio anterior, esto es, en las dos primeras hojas los sujetos debían cumplimentar los datos personales (v.g.: sexo, edad, etc.), y a continuación se presentaban las instrucciones para la realización del cuestionario, junto con 3 ejercicios cumplimentados a modo de ejemplos para familiarizarles con la tarea.

Las siguientes hojas del cuadernillo ofrecían, en el margen superior, la escala evaluativa (0-8 puntos) que sirve de guía para la emisión 'de los juicios, y seguidamente, la serie de enunciados (en el margen izquierdo) encabezados con giros autorreferenciales, con sus correspondientes escalas numéricas (en el margen derecho) donde los sujetos reflejaban sus juicios. Cada frase debía ser juzgada en función del grado de acuerdo entre las ideas del padre o madre que realiza el cuestionario y la que se refleja en cada enunciado.

La recogida de la información se realizó en diversos centros escolares a los que acudían los padres de forma voluntaria. Se hizo gran hincapié en señalarles que en la prueba no existían aciertos ni errores, tan sólo debían reflejar su opinión personal acerca de las diferentes frases, tomando como criterio evaluador la escala numérica que se les había explicado en las instrucciones. El tiempo de ejecución de la tarea era ilimitado.

\section{Resultados}

Los resultados se expondrán en bloques diferentes según el tipo de objetivo en estudio y el análisis estadístico requerido para cada propósito.

\section{Fiabilidad del cuestionario}

Se realizó este análisis mediante el paquete estadístico SPSS, obteniéndose unos coeficientes de fiabilidad satisfactorios para cada escala, los cuales presentamos en la Tabla II. 
TABLA II

Coeficientes de fiabilidad para cada escala del cuestionario de las teorias implícitas de educación

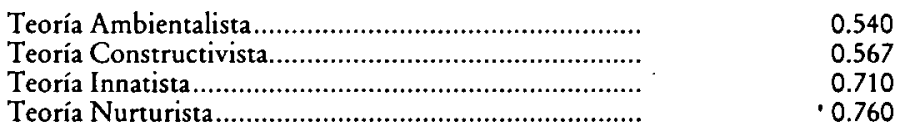

2. Grado de acuerdo entre las teorias sustentadas por el padre y la madre

Se realizó un análisis de correlación canónica mediante el programa P6M del paquete estadístico BMDP, para estudiar el grado de coincidencia entre las teorías educativas sustentadas por ambos cónyuges. Para ello se tomaron las puntuaciones medias, en las distintas teorías, obtenidas por los miembros de las 72 parejas participantes.

Se obtuvo una sola correlación canónica de 0.615 , altamente significativa $(p \leqslant .0001)$, lo que indica que las teorías que comparten los padres varones coinciden en alto grado con las de las madres. No obstante, no existe una total correspondencia entre las preferencias de ambos padres. Analizando los coeficientes de estructura de ambas variables canónicas, se observa un perfil que indica la relación fundamentalmente entre las teorías innatista, ambientalista y constructivista en los padres con las teorías innatista y ambientalista de las madres.

\section{Distribución de las teorías de los padres en función de las variables demográficas estudiadas}

Con el fin de estudiar las variables demográficas que sirven como índices diferenciadores en la elección de las distintas teorías, utilizamos un procedimiento novedoso que describimos a continuación.

a) Determinación de las dimensiones de clasificación respecto al grupo de padres extremos: En primer lugar se elaboraron los índices de polaridad para cada teoría en estudio, que nos indican el grado de preferencia exclusiva de los padres hacia una teoría en particular. La polaridad se obtiene a partir de la fórmula ideada para tal fin en el estudio normativo (Triana y Rodrigo, 1985). Estos índices fueron transformados posteriormente en puntuaciones típicas, en función de las cuales seleccionamos para el estudio los sujetos localizados en el $30 \%$ inferior y $30 \%$ superior en la curva normal. Tomando esta muestra de sujetos extremos intentábamos localizar las dimensiones que clasifican a estos padres, y después, extrapolar estos resultados a la muestra total. Para ello iniciamos la búsqueda de las teorías compartidas y rechazadas en esta muestra de sujetos. Así; establecimos conjuntos de teorías combinándolas primero de dos en dos, luego en grupos de tres y por último atendiendo a la combinación de las cuatro teorías. Al final tomamos aquellas combinaciones en las que el número de padres adscritos fuese superior al que pudiera salir por puro azar.

Concretamente, se localizaron los tres conjuntos siguientes:

Grupo 1. Padres que aceptan las teorías ambientalista y constructivista y rechazan la nurturista e innatista ( $\mathrm{N}=19$ sujetos). 
Grupo 2. Padres que aceptan las teorías innatista y constructivista y rechazan la ambientalista y nurturista ( $\mathrm{N}=10$ sujetos).

Grupo 3. Padres que aceptan las teorías innatista y nurturista y rechazan la ambientalista y constructivista ( $\mathrm{N}=8$ sujetos).

En estos tres grupos quedaron clasificados un total de 37 sujetos extremos. Seguidamente se realizó un análisis discriminante, mediante los paquetes estadísticos SPSS, para examinar las diferencias entre los grupos. En esta ocasión se tomaron como grupo los tres conjuntos de padres formados y como variables discriminantes las teorías. Se pretendía con ello encontrar el número de dimensiones necesarias para describir la separación entre los diferentes grupos de padres encontrados, según las teorías que aceptan y rechazan. Los resultados se presentan a continuación.

TABLA III

Función Discriminante Canónica

\begin{tabular}{rcccccccc}
\hline $\begin{array}{c}\text { Valor } \\
\text { propio }\end{array}$ & $\begin{array}{c}\% \text { de } \\
\text { Varianza }\end{array}$ & $\begin{array}{c}\% \\
\text { Acum. }\end{array}$ & $\begin{array}{c}\text { Correlación } \\
\text { Canónica }\end{array}$ & $\begin{array}{c}\text { Después } \\
\text { de Función }\end{array}$ & $\begin{array}{c}\text { Lambda } \\
\text { de Wilks }\end{array}$ & $\begin{array}{c}\text { CHI- } \\
\text { Cuadrado }\end{array}$ & D.F. & $\begin{array}{c}\text { Signi- } \\
\text { ficación }\end{array}$ \\
\hline 14.3656 & 86.68 & 86.68 & .9669 & 0 & .0203 & 128.619 & 6 & .0001 \\
2.2072 & 13.32 & 100.00 & .8296 & 1 & .3118 & 38.459 & 2 & .0001 \\
\hline
\end{tabular}

Se obtienen dos funciones discriminantes altamente significativas $(p \leqslant .0001)$, donde la primera explica unas seis veces más varianza que la segunda. El valor de la Lambda de Wilks, teniendo en cuenta todas las funciones discriminantes es de .0203 , y de .3118 si tenemos en cuenta sólo la segunda función. Estos resultados muestran una gran separación entre los grupos estudiados. A continuación presentamos otros datos de interés para el análisis de los resultados (ver Tabla IV).

\section{TABLA IV}

Coeficientes de estructura

\begin{tabular}{|c|c|c|}
\hline & Función 1 & Función 2 \\
\hline 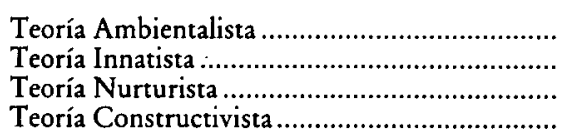 & $\begin{array}{r}.6985^{*} \\
-.6733^{*} \\
-.3274 \\
.2917\end{array}$ & $\begin{array}{r}-.0518 \\
.0890 \\
-.8316^{*} \\
.7736^{*}\end{array}$ \\
\hline
\end{tabular}

A través de los coeficientes de estructura se observa que la primera función discriminante se identifica con las teorías ambientalista e innatista, presentándose como opuestas en esta dimensión, mientras que en la segunda función las teorías que se identifican con ella son la nurturista y la constructivista, y se presentan también altamente polarizadas. Un resultado importante fue que el $100 \%$ de los sujetos estudiados quedaron perfectamente clasificados en estas dos funciones discriminantes.

b) Aplicación de las dos funciones discriminantes obtenidas al conjunto total de padres: El segundo paso consistió en clasificar al total de la mues- 
tra de padres según las dimensiones encontradas en los padres extremos. Para ello realizamos un nuevo análisis discriminante tomando las teorias (en puntuaciones de polaridad) como variables discriminantes y como grupo las funciones discriminantes anteriormente obtenidas. Los resultados se presentan a continuación.

TABLA V

Función Discriminante Canónica

\begin{tabular}{rcccccccc}
\hline $\begin{array}{c}\text { Valor } \\
\text { propio }\end{array}$ & $\begin{array}{c}\% \text { de } \\
\text { Varianza }\end{array}$ & $\begin{array}{c}\% \\
\text { Acum. }\end{array}$ & $\begin{array}{c}\text { Correlación } \\
\text { Canónica }\end{array}$ & $\begin{array}{c}\text { Después } \\
\text { de Función }\end{array}$ & $\begin{array}{c}\text { Lambda } \\
\text { de Wilks }\end{array}$ & $\begin{array}{c}\text { CHI- } \\
\text { Cuadrado }\end{array}$ & D.F. & $\begin{array}{c}\text { Signi- } \\
\text { ficación }\end{array}$ \\
\hline 2.2149 & 81.97 & 81.97 & .8300 & 0 & .2091 & 574.277 & 6 & .0001 \\
.4873 & 18.03 & 100.00 & .5724 & 1 & .6723 & 145.695 & 2 & .0001 \\
\hline
\end{tabular}

Se obtienen dos funciones discriminantes altamente significativas $(p \leqslant .0001)$, donde la primera explica unas cuatro veces más varianza que la segunda. El valor de la Lambda de Wilks, teniendo en cuenta todas las funciones discriminantes es de .2091 , y de .6723 si tenemos en cuenta sólo la segunda función. Estos resultados nos indican que existen nuevamente diferencias entre los grupos de padres estudiados. Veamos a continuación los coeficientes de estructura (Tabla VI).

TABLA VI

Coeficientes de estructura

\begin{tabular}{|c|c|c|}
\hline & Función 1 & Función 2 \\
\hline 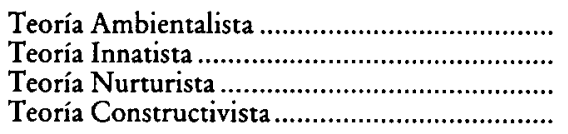 & $\begin{array}{l}-.7365 * \\
.6313^{*} \\
.0609 \\
.0578\end{array}$ & $\begin{array}{l}-.0362 \\
-.0355 \\
.8885 * \\
-.7633^{*}\end{array}$ \\
\hline
\end{tabular}

Estos resultados coinciden con los de la fase anterior, esto es, la clasificación de los padres se realiza a través de dos dimensiones que contraponen la primera, la teoría ambientalista con la innatista y la segunda, la nurturista con la constructivista. En esta ocasión el porcentaje de sujetos que quedó bien clasificado fue el $93,80 \%$, proporción que, como se puede observar, es bastante alta.

c) Clasificación de los grupos de padres en función de las variables demográficas analizadas: El último paso consistió en realizar un análisis discriminante tomando como grupo los distintos grupos de padres en función de las teorias que eligen y rechazan y como variables discriminantes las variables demográficas, nivel profesional, nivel de estudios y zona de residencia. La edad de los padres y el número de hijos no se incluyó en este estudio porque en los análisis discriminantes exploratorios no parecían contribuir a la separación entre los grupos de padres. Los resultados se muestran a continuación (Tabla VII).

Se obtiene una función discriminante altamente significativa $(p \leqslant .0001)$,

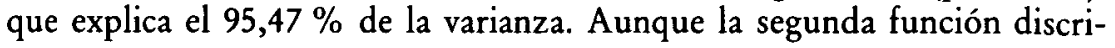


TABLA VII

Función Discriminante Canónica

\begin{tabular}{rrrcccccc}
\hline $\begin{array}{c}\text { Valor } \\
\text { propio }\end{array}$ & $\begin{array}{c}\% \text { de } \\
\text { Varianza }\end{array}$ & $\begin{array}{c}\text { \% } \\
\text { Acum. }\end{array}$ & $\begin{array}{c}\text { Correlación } \\
\text { Canónica }\end{array}$ & $\begin{array}{c}\text { Después } \\
\text { de Función }\end{array}$ & $\begin{array}{c}\text { Lambda } \\
\text { de Willss }\end{array}$ & $\begin{array}{c}\text { CHI- } \\
\text { Cuadrado }\end{array}$ & D.F. & $\begin{array}{c}\text { Signi- } \\
\text { ficación }\end{array}$ \\
\hline .0870 & 95.47 & 95.47 & .2828 & 0 & .9162 & 32.112 & 6 & .0001 \\
.0041 & 4.53 & 100.00 & .0641 & 1 & .9959 & 1.512 & 2 & .4695 \\
\hline
\end{tabular}

minante no llegue a obtener significación estadística, por tratarse esta investigación de un estudio exploratorio, hemos considerado conveniente utilizar también la información que ésta nos ofrece, ya que, como veremos a continuación, contribuye en alto grado a la diferenciación de los grupos de padres respecto a las variables demográficas. No obstante, las conclusiones que de ellas se deriven deberán ser interpretadas con cierta cautela. En cuanto a los coeficientes de estructura, los resultados se presentan a continuación (Tabla VIII).

TABLA VIII

Coeficientes de estructura

\begin{tabular}{|c|c|c|}
\hline & Función 1 & Función 2 \\
\hline Variable Nivel profesional.. & $.96282 *$ & -.00761 \\
\hline 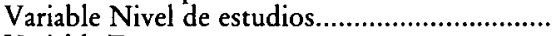 & $.76501^{*}$ & .35898 \\
\hline 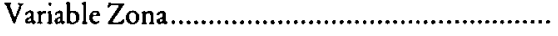 & -.13923 & $.98208 *$ \\
\hline
\end{tabular}

En esta ocasión, la primera función discriminante se identifica con las variables «nivel profesional y nivel de estudios", mientras que la segunda función lo hace con la variable «zona». Veamos a continuación cómo se distribuyen los grupos de padres en función de estas dos dimensiones (Tabla IX).

\section{TABLA IX}

Tipo de teorias de los padres $y$ su distribución en función de las variables demográficas

\begin{tabular}{clcc}
\hline $\begin{array}{c}\text { Grupo } \\
\text { Padres }\end{array}$ & \multicolumn{1}{c}{$\begin{array}{c}\text { Descriptores } \\
\text { Sociales }\end{array}$} & Asumen & Rechazan \\
\hline 1 & $\begin{array}{l}\text { - Alto nivel educativo y } \\
\text { profesional } \\
\text { Zona urbana }\end{array}$ & $\begin{array}{c}\text { Ambientalista-Cons- } \\
\text { tructivista }\end{array}$ & Nurturista-Innatista \\
2 & $\begin{array}{l}\text { Alto nivel educativo y } \\
\text { profesional }\end{array}$ & $\begin{array}{c}\text { Innatista-Constructivis- } \\
\text { ta }\end{array}$ & $\begin{array}{c}\text { Ambientalista-Nurtu- } \\
\text { rista }\end{array}$ \\
3 & $\begin{array}{l}\text { Bajo nivel educativo y } \\
\text { profesional }\end{array}$ & Innatista-Nurturista & $\begin{array}{c}\text { Ambientalista-Cons- } \\
\text { tructivista }\end{array}$ \\
\hline
\end{tabular}

Se observa que los padres con bajo nivel profesional y de estudios suelen asumir las teorías innatista y nurturista en mayor grado que los de alto nivel, que tienden a compartir las teorías ambientalista y constructivista, y 
en menor grado las innatistas. Asimismo, se observa esta misma pauta con respecto a la variable zona, de forma que los padres que residen en los ambientes rurales tienden a compartir las teorías innatista y nurturista frente a los de la zona urbana que asumen la ambientalista y la constructivista.

\section{Discusión y conclusiones}

Abordar el campo de las concepciones sobre la educación y el desarrollo supone introducirnos en el estudio de los padres para analizar el conjunto de ideas que soportan su comprensión de las situaciones educativas, y en última instancia, la selección de las prácticas de crianza que utilizan. Este acercamiento sería irreflexivo si planteáramos este análisis atendiendo a conjuntos de información estáticos, sin tomar en cuenta los distintos valores, atribuciones, sentimientos, etc., que comparten los padres, y que en definitiva, intervienen en el procesamiento de la información. Por esta razón, es necesario atender a un análisis comparativo entre conocimiento y creencia (Sigel, 1985), sin negar, por supuesto, la gran relación existente entre ambos conceptos.

En este sentido, denominamos "conocimiento" al conjunto de ideas que reciben los sujetos del amplio bagaje informativo que ofrece la cultura a la que pertenecen. Así, las teorías, entendidas como conocimiento, presentan límites difusos ya que pueden ser activadas simultáneamente sin detrimento de ninguna de ellas, ya que no llevan implícito un carácter de verdad que haga polarizarse a los sujetos (Triana y Rodrigo, 1985). Por el contrario, el término "creencia» implica un conocimiento asumido y cargado de factores emocionales, que predispone a los sujetos a interpretar la realidad de forma altamente subjetiva y discretizada, tomándola como guía para realizar evaluaciones, hasta el punto de negar evidencias tangibles que no la corroboren. De este modo, la cultura sirve de sustento para el desarrollo del conocimiento humano, y de entre todo este cúmulo informativo, los padres se decantan por determinadas teorías educativas que toman como guía para su acción. La responsabilidad de esta selección recae en los contextos sociales próximos en los que se desenvuelven los sujetos. Estos contextos facilitan que los padres se hayan visto expuestos a un mayor cúmulo de experiencias relativas a algunas teorías frente a otras. Así, por ejemplo, a través de nuestras vivencias como hijos obtenemos información sobre las teorías asumidas por nuestros propios padres; el tipo de enseñanza que recibimos en la escuela suele ser similar a la de nuestros padres puesto que éstos eligen los colegios acordes a sus criterios educativos; en muchas ocasiones, nuestros compañeros de juego pertenecen a familias con pautas de crianza similares a las de nuestros padres, etc. Este cúmulo de información sobre determinadas teorías suele venir acompañado de un conjunto de valores y atribuciones compartidos por estos grupos sociales, lo que hace que se conviertan en verdaderos filtros interpretativos de la realidad educativa, llegando a adquirir un valor de creencia.

Otro de los contextos que contribuyen a la formación de las creencias es la unidad familiar. En nuestro estudio se obtuvo una correlación canónica de .615 (altamente significativa), lo cual demuestra un gran acuerdo entre las parejas respecto a las ideas que tienen sobre la educación y el desarrollo de sus hijos. Esta relación se establece fundamentalmente entre las 
puntuaciones dadas por los padres varones a las teorías innatista, ambientalista y constructivista con las de las ambientalista e innatista de las madres. Algunos autores (Wegner y Vallacher, 1977; McGillicuddy-De Lisi, 1982) afirman que el intercambio de experiencias e ideas que realizan los esposos acerca de sus hijos, produce la aproximación de sus concepciones. De este modo, el ambiente familiar proporciona uno de los contextos claves para la formación de experiencias cuya síntesis constituirá las creencias de los padres.

Un resultado interesante ha sido el tipo de creencias que asumen los padres. No se han encontrado padres "puros", sino que, por el contrario, éstos suelen compartir más de una teoría. Así por ejemplo, se obtuvieron tres tipos de padres: a) Aquéllos que asumen ideas ambientalistas-constructivistas; b) otros que comparten ideas innatistas-constructivistas, y c) aquellos que asumen ideas innatistas-nurturistas. De este modo las creencias de los padres se distribuyen atendiendo a dos dimensiones de clasificación, la primera definida por las teorias innatista y ambientalista que toman posiciones altamente polarizadas, y la segunda, definida por las teorías nurturista y constructivista, también con valores contrapuestos.

La primera dimensión puede interpretarse como el grado de influencia percibida por los padres respecto a sus hijos. Así, una teoría innatista implica una concepción pasiva del-rol que tienen los progenitores y el ambiente en general en el desarrollo de los niños, dejando el papel preponderante a la herencia (Antill, 1987; Mugny y Carugati, 1985). Por el contrario, una teoría ambientalista comparte ideas de cambio motivado por factores ambientales, lo que enfatiza el papel de los padres en el desarrollo de sus hijos. Por su parte, la segunda dimensión hace referencia a cómo reciben los padres al propio niño, variando desde una concepción muy simplista y poco articulada del desarrollo contemplada en la teoría nurturista (v.g.: importancia de la alimentación, salud, etc.), hasta una concepción más compleja propia de la teoría constructivista (Newberger, 1980; Sameroff y Feil, 1985).

En definitiva, los padres son capaces de representarse mentalmente las diferentes teorías, y lo que es más importante, suelen estar de acuerdo con mezclas que en todos los casos muestran una gran coherencia conceptual ya que se establecen entre las teorías más afines y nunca entre las contradictorias. Por ejemplo, los padres ambientalistas muestran gran desacuerdo con las ideas innatistas. Asimismo, cuando un padre asume una teoría nurturista, que se fundamenta en una concepción poco evolutiva del niño ya que la 'buena alimentación y el ejercicio son factores beneficiosos a cualquier edad, no suele, a su vez, ser partidario de la teoría constructivista cuyos presupuestos defienden el trato diferencial a medida que el niño crece.

Pasamos ahora a comentar el papel de algunas variables demográficas en la elección de las teorías que asumen los padres. El análisis discriminante mostró dos dimensiones de clasificación. La primera se identifica con el nivel profesional y de estudios de los padres, y la segunda con la zona de residencia. Así, los resultados muestran que los padres que consideran la dotación genética como máximo responsable de la evolución de los niños, y que resaltan la importancia de la salud, el deporte y la alimentación para conseguir un buen desarrollo, son aquellos que presentan bajos niveles profesionales y de estudios. Asimismo, estas concepciones son prioritariamen- 
te compartidas por los padres que residen en las zonas rurales frente a los urbanos."

Por el contrario, los padres de niveles profesionales medios y altos, así como los que residen en zonas urbanas, valoran en mayor grado la importancia que tiene el medio sobre el desarrollo de los niños, sintiéndose más protagonistas y responsables de su educación, aunque en algunos casos no niegan terminantemente las limitaciones que pueda imponer la herencia. A su vez, estas ideas están matizadas por la consideración de que el niño, por sus propios medios, puede también contribuir a su desarrollo. De este modo, les ven como seres competentes y capaces que pueden actuar modificando los efectos ambientales. Estos resultados coinciden con los obtenidos por otros autores (Ninio, 1979; Zukow, 1984; Schaefer y Edgerton, 1985; Palacios, 1986).

Por su parte, la edad de los padres o el número de hijos no parece influir en la selección de las teorías que asumen los padres. No obstante, Knight (1983) encontró que las familias que tienen pocos hijos son más innatistas que las familias más numerosas. Probablemente, la utilización de grupos de padres con mayor contraste en los niveles de estas variables puedan aportar, en investigaciones futuras, mayor luz sobre la influencia de las mismas.

En definitiva, el sistema de creencias de los padres sobre la educación y el desarrrollo de los hijos, no es un mero reflejo del amplio conjunto de «mensajes culturales». Tampoco es el producto de la construcción personal e idiosincrásica realizada por los sujetos a partir de sus experiencias directas. Ambos factores, cultura y cognición, son interdependientes, ya que los padres elaboran sus teorias a partir de las ideas que aporta la cultura, y que llega a ellos a través de los intercambios normativizados que se realizan en los contextos sociales próximos. En definitiva, la regularidad que ofrece la cultura queda mediatizada por la construcción individual que es la responsable de las mezclas coherentes de teorías que se observan en los padres de los distintos grupos sociales.

\section{Referencias}

ANTILL, J. K. (1987). Parents' beliefs and values about sex roles, sex differences, and sexuality: Their sources and implications. En P. Shaver y C. Hendrick (eds.). Sex and gender: Review of personality and social psychology. Vol. 7, 294-326. Beverly Hills, C.A.: Sage.

BACON, M. K., y ASHMORE, R. D. (1986). A consideration of the cognitive activities of parents and their role in the socialization process. En R. Ashmore y D. Brodzinsky (eds.). Thinking about the family: Views of parents and children. Nueva Jersey: Lawrence Erlbaum Associates.

BROOKS-GUNN, J. (1986). Maternal beliefs about children's sex-typed characteristics as they relate to maternal behaviour. En I. E. Sigel (ed.). Parental belief Systems. The psychological consequences for children. Nueva Jersey: Lawrence Erlbaum Associates.

CASHMORE, J. A., y GoODNOw, J. J. (1985). Agreement between generations: A two-process approach. Child Development. 56, 493-501.

GOODNOw, J. J. (1987). Blending developmental and social approaches to adult social cognition: The case of parents' ideas, actions and feelings. Paper presented at the meeting of the Society for Research in Child Development. Baltimore.

KELLY, G. (1955). The psychology of personal constructs. Vols. I y II. Nueva York: Norton.

KNIGHT, R. (1983). Parents' ideas about childhood and parenting: A constructivist approach. Unpublished Ph. D. Thesis, MacQuarie University, North Ryde, Australia.

MCGILLICUDDY-DE LISI, A. (1982). The relation between family configuration and parental beliefs about child development. En L. M. Laosa e I. E. Sigel (eds.). Families as learning environments for children. Nueva York: Plenum Press. 
Mugny, G., y Carugati, F. (1985). L'intelligence au pluriel: Les répresentations sociales de l'intelligence et de son développement. Cousset: Etitions Delval.

NEWBERGER, C. M. (1980). The cognitive structure of parenthood: Designing a descriptive measure. En R. L. Selman y R. Yando (eds.). New directions for child development. Vol. 7, 45-67. San Francisco: Jossey-Bass.

NiNIO, A. (1979). The naive theory of the infant and other maternal attitudes in two subgroups in Israel. Child Development. 50, 976-980.

PalaCios, J. (1987). Las ideas de los padres sobre sus hijos en la investigación evolutiva. Infancia y Aprendizaje, 39-40, 97-111.

RoDRIGO, M. J. (1985). Las teorías implícitas en el conocimiento social. Infancia y Aprendizaje, 31-32, 145-156.

SAMEROFF, A. J., y FEIL, L. A. (1985). Parental concepts of development. En I. E. Sigel (ed.). Parental belief systems. The psychological consequences for children. Nueva Jersey: Lawrence Erlbaum Associates.

SCHAEFER, E., y EDGERTON, M. (1985). Parent and child correlates of parental modernity. En I. E. Sigel (ed.). Parental belief systems. The psychological consequences for children. Nueva Jersey: Lawrence Erlbaum Associates.

SIGEL, I. E. (1985). A conceptual analysis of beliefs. En I. E. Sigel (ed.). Parental belief systems. The psychological consequences for children. Nueva Jersey: Lawrence Erlbaum Associates.

SIGEL, I. E. (1986). Reflections on the belief-behavior connection: Lessons learned from a research program on parental belief systems and teaching strategies. En R. Ashmore y D. Brodzinsky (eds.). Thinking about the family: Views of parents and children. Nueva Jersey: Lawrence Erlbaum Associates.

SKINNER, E. A. (1985). Determinants of mother sensitive and contingent-responsive behavior: The role of childrearing beliefs and socio economic status. En I. E. Sigel (ed.). Parental belief systems. The psychological consequences for children. Nueva Jersey: Lawrence Erlbaum Associates.

Triana, B., y RoDrigo, M. J. (1985). El concepto de infancia en nuestra sociedad: Una investigación sobre teorías implicitas de los padres. Infancia y Aprendizaje, 31-32, 157-171.

WEGNER, D. M., y VALLACHER, R. R. (1977). Implicit Psychology: An introduction to social cognition. Nueva York: Oxford University Press.

ZUKOW, P. G. (1984). Folk theories of comprehension and caregiver practices in a rural-born population in central Mexico. Manuscrito no publicado.

\section{Extended Summary}

The idea of parents as people who simply react to their children's overt behaviour is out dated. At present, they are seen as thinking individuals confronting the task of educating their children equipped with an ample stock of knowledge on childhood. Assuming this hypothesis, the necessity for a theoretical model explaining the origins of parents' conceptions on education and development is obvius (Sigel, 1985; Goodnow, 1985, 1987; Nespor, 1987). The aim of this paper is to analyse the sources which form the basis from which these theories are elaborated an how these are formed in parents' mind.

Goodnow (1987) has reviewed the two theoretical approaches which have been used to address this subject. On the one hand, the psychological approach considers these theories to be the product of a personal construction which parents achieve based on their background of episodic experiences. On the other hand, the sociological approach places the responsability for the construction of such conceptions once again on culture and society.

In our opinion, both views present serious limitations. Thus, we consider it necessary to propose a new approach entitled «social-constructivist” which analyses interactively the influence of both of these factors, culture and cognition. We hypothesize that parents construct personal theories from their episodic experiences whose content and mode of acquisi- 
tion (through the social interaction) have a cultural origin. Regarding the content of parents' educational theories, Triana and Rodrigo (1985) demonstrated the existance of conceptions in parents' conceptual system with deep cultural roots. This fact shows the effect of culture on the knowledge of the lay-man. Nevertheless, the specific type of theory which parents adopt out of the variety of conceptions that they encounter has yet to be studied together with the role of close social contexts in the adoption of a particular conception.

With respect to the type of theories which parents adopt, we have found that they are never an exact copy of cultural theories. On the contrary, they are the product of a personal elaboration based on cultural contents. Hence parents adopt coherent mixtures of theories which share common features rather than pure ones. Three main types of mixed belief groupings have been found: first, parents who accept environmentalist and constructivist ideas and reject nurturist and nativist ones; second, parents who accept nativist and constructivist ideas and reject environmentalist and nurturist ones; and third, parents who accept nativist and nurturist ideas and reject environmentalist and construtivist ones. In this way, parents who are environmentalists always disagree with nativists ideas. Likewise, when parents assume a nurturistic theory based on the importance of childrens' nourishment and health, without any distinction in age, they are not usually in favour of the constructivist theory whose assumptions defend providing differential treatment to the chlid as he/she grows.

As far as the role of close social contexts in selecting theories which parents assume, it was observed that out of all the indicators analysed, parents' academic and professional background together with the area in which they. live define these critical interactive contexts which determine the mixture of theories that parents assume. 